Aus dem zoologischen Institut der Universität Berlin.

\title{
Über ein bemerkenswertes Strukturelement (Heterochromosom?) in der Spermiogenese des Menschen.
}

Von

Dr. S. Gutherz.

Hierzu Tafel VI und 2 Textfiguren.

Seitdem vor nunmehr zwanzig Jahren Henking durch seine grundlegenden Beobachtungen an Pyrrhocoris apterus die Lehre von den Heterochromosomen inaugurierte, hat unsere Kenntnis dieser Gebilde, namentlich im letzten Jahrzehnt, einen gewaltigen Umfang angenommen. Das hohe Interesse, welches man dem jüngsten Zweige der Zellkernforschung von verschiedenen Seiten eutgegenbrachte, erklïrt sich als ein zwiefaches. Einmal gelang es auf diesem Wege, zwischen den Chromosomen ein und desselben Zellkernes höchst bedeutsame Differenzierungen aufzudecken, sodann eröffnete sich zum ersten Male die Möglichkeit, morphologisch scharf charakterisierte Bestandteile des Kernes mit einer wichtigen Lebenserscheinung in funktionelle Beziehung zu setzen. Nur gewisse Typen der bereits in mehrfachen Erscheinungsformen bekannt gewordenen Heterochromosomen kommen in der letzterwăhnten Hinsicht in Betracht, nämlich die von Boveri so genanuten Geschlechtschromosomen. Für diese Chromosomenformen hatte man bereits seit mebreren Jahren angenommen, dass ihre verschiedene Verteilung auf die Spermien den gleichzeitig festgestellten verschiedenen Chromatinbestand der männlichen und weiblichen Individuen bedinge, neuerdings ist der direkte Nachweis bierfür von Morrill (bei Hemipteren) und von Gulick (bei Nematoden) durch Ermittelung eines fast lückenlosen Chromatinzyklus erbracht worden. Damit aber ist eine, wenn auch noch nicht als kausal erwiesene, Beziehung der Heterochromosomen zur Geschlechtsdifferenzierung sichergestellt. ${ }^{1}$ )

1) Näheres über den gegenwärtigen Stand der HeterochromosomenForschung findet sich in Wils ons (1911 b) and des Verfassers (1911) zusammenfassenden Darstellungen, welch letztere die Frage nach einem etwaigen kausalen Zusammenhange zwischen Heterochromosomen und Geschlechtsdifferenzierung besonders eingehend behandelt. 
Bis vor kurzem waren Geschlechtschromosomen nur im Tierkreise der Arthropoden, insbesondere bei Insekten bekannt. ${ }^{\text {) }}$ Es erregte daher ein besonderes Interesse, als man in jüngster Zeit auch bei einer Reibe von Nematoden typische Geschlechtschromosomen nachzuweisen vermochte, und es konnte die Hoffnung entstehen, hier einer Erscheinung. von allgemeinerer Verbreitung auf die Spur gekommen zu sein. So lag es nabe, neue Tiergl'uppen zur Untersuchung heranzuzielen, darunter auch die Vertebraten. Rasch wurden denn auch bei letzteren positive Befunde erhoben (Guyer, v. Winiwarter und Saintmont, Jordan, Stevens). Betrachtet man indessen die Abbildungen, welche von den Autoren ihren Mitteilungen beigegeben werden, so kann man sich in manchen Fällen des Eindruckes nicht erwehren, dass bei den höheren Wirbeltieren die ron den Insekten her gewohnte, mitunter schematische Klarheit der Chromatinverhältnisse öfters vermisst wird. Diese Bemerkung gilt auch für Guyers letzte Publikation, die das wichtigste Objelit, dem wir unsere Arbeit widmen können, den Venschen, betrifft und bei ihm Geschlechtschromosomen konstatiert. ${ }^{2}$ )

Je wichtiger aber der Gegenstand einer Untersuchung ist, um so kritischer soliten wir bei der Feststellung der Tatsachen

1) Für zwei Echinidenarten hat Baltze r (1909) Chromatinelemente beschrieben, die an die gepaarten Geschlechtschromosomen der Insekten erinnern; ihre verschiedene Verteilung in der Oogenese soll einen Chromatindimorphismus der reifen Eier bedingen. Dieser Fall steht in der Literatur bisher vereinzelt da, während sonst ein derartiger Dimorphismus stets an den Spermien beobachtet wird.

2) G n yers (1910) Darstellung seiner Befunde am Menschen ist kurz die folgende. Die Chromosomenzahl der Spermiogonie beträgt 22. Im Kern der Spermiocyten finden sich zwei verschieden grosse, voneinander entfernt liegende chromatische Körper, die der Autor auf zwei Chromosomen der Spermiogonie zurïckführt. In der ersten Reifungsmitose legen sich diese beiden Chromosomen zu einem die Zusammensetzung aus zwei verschieden grossen Komponenten noch zeigenden Gebilde zusammen und dieses Gebilde erfährt Heterokinese. So entstehen schliesslich zwei Sorten von Spermiden, deren eine im Kern wieder die beiden verschieden grossen chromatischen Körper getrennt zeigt. während die andere frei von ihnen ist. Die Chromosomenzahl in der Metaphase des Spermiocyten beträgt 12 (10 bivalente gewöhnliche Chromosomen und 2 Heterochromosomen), die Praespermidenmitose zeigt 5 bezw. 7 Chromosomen, was sich nach Guyer daraus erklärt, dass eine nochmalige Konjugation der gewöhnlichen Chromosomen stattgefunden hat. 
und ihrer Deutung verfahren. Ich ergriff daher gern die sich mir bietende Gelegenheit, selbst menschliches Material zu untersuchen und die Angaben Guyers einer Nachprüfung zu unterzieben. Sind meine Resultate auch noch leine abschliessenden, so erscheint, wie ich meine, ihre Mitteilung im Hinblick auf das hohe Interesse des Gegenstandes berechtigt; auch sehe ich eine wesentliche Aufgabe der vorliegenden Publikation darin, die Anlegung $z u$ weiteren Untersuchungen $z u$ gebell, da bei einem so schwierigen und so schwer $z u$ beschaffenden Objekt erst das Zusammenwirken verschiedener Forscher ein gesichertes Endelgebnis herbeifübren dürfte.

\section{Material und Technik der Untersuchung.}

Das Hauptmaterial dieser Arbeit stammt von einem chirurgischen Falle (23jabriger Mann) und wurde von Herrn Professor H. Poll (Berlin), der es mir zur Untersuchung der hier behandelten Frage freundiichst überliess, lebenswarm in die Fixierungsmittel (Flemmings starkes Gemisch und Zenkersche Flüssigkeit) eingelegt. Ich möchte nicht verfehlen, Herm Professor Poll auch an dieser Stelle für die Überlassung des kostbaren Objektes meinen herzlicbsten Dank auszusprechen. Diesen Hoden, welcher sich als in lebhaftester Entwicklung befindich und volzüglich fixiert erwies, werde ich im folgenden als Fall A bezeichnen.

Meinen verbindlichsten Dank sage ich sodann Herrn Professor Fr. Me ves (Kiel), der mir, durch gütige Vermittelung von Herrn Professor P.o11, Hodenstücke dreier Justifizierter (in F l e m m in g s starkem Gemisch fixiert) liebenswürdiger Weise zusandte. Ich erhielt so ein ausserordentlich wertvolles Ergänzungsmaterial (Fälle BI-III). Sümtliche drei Stücke sind ausgezeichnet fixiert; Fall BI zeigt lebhafteste Spermiogenese, auf das Verbaiten der beiden anderen Fälle wird noch zurückzukommen sein.

Als Färbungsmethode diente mir vorzugsweise Eisenhämatoxylin nach M. Heidenhain, ferner kamen das Biondische Gemisch, das nur am Z enker-Material, also lediglich am Fall A. angewandt werden konnte, und die Flemming sche Dreifarbenmethode zur Benutzung. Die Schnittdicke betrug 5 oder $10 \mu$. Bei der Untersuchung der Präparate leistete mir die neue Zeisssche Mikro-Nernstlampe vortreffliche Dienste. 


\section{Methodologische Vorbemerkungen.}

Ein völlig sicherer Nachweis jener Chromosomenformen, die wir wegen ihres abweichenden Verhaltens als Heterochromosomen bezeichnen, ist nur dann möglich, wenn es gelingt, die zusammenhängende Geschichte der Chromatinverhältnisse der betreffenden Stadien, insbesondere auch die genaue Chromosomenzahl wăhrend derselben zu verfolgen. Es sei hervorgehoben, dass dieses Postulat bei einer grossen Reihe von Insekten, die ja die typischsten Beispiele von Heterochromosomentrăgern bilden, erfüllt ist. Da nun der Mensch, wie von vornherein gesagt sei, keineswegs zu jenen günstigen Chromatinobjekten gehört, vielmehr schon eine Ermittelung seiner Chromosomenzahl bei dem gegenwärtigen Stande unserer Technik auf ausserordentliche Schwierigkeiten stösst, so müssen wir, wenn wir hiel sozusagen die Diagnose auf Heterochromosomen stellen wollen, unsere Zuflucht zu gewissen, weniger sicheren Kriterien nehmen, die wiederum im einzelnen von verschiedener Wertigkeit sind. Ein gutes Hilfsmittel zur Erkennung eines Heterochromosoms kann unter Umständen seine in der Metaphase der Hitose hervortretende eigentümliche Gestalt abgeben, aber dieses Vorkommnis ist nur bei wenigen Objekten (z. B. den Grylliden) beobachtet und kommt für unser Objekt nicht in Betracht. Wichtiger ist die bei der Geschlechtszellenbildung (namentlich der Spermiogenese) in einer der Reifungsteilungen hïufig beobachtete Erscheinung der Heterokinese, die darin besteht, dass ein Chromosom (bezw. ein Chromosomenkomplex) ungeteilt bleibt und in nur eine Tochterzelle übergeht oder dass bei der T'eilung zwei ungleich grosse Chromosomen (bezw. Chromosomenkomplexe) voneinander getrennt werden und verschiedenen Polen zustreben. ${ }^{1}$ ) Die einwandfreie Beobachtung einer Heterokinese darf, auch bei nicht genau bekannter Gesamtchromosomenzahl, als ausreichend für den Nachweis eines oder mehrerer Heterochromosomen gelten. Ein weniger sicheres Krriterium stellt die sogenannte Heteropyknose ${ }^{2}$ ) dar: sie aussert sich in einem verschiedenen Konzen-

1) Ich fasse den Begriff der Heterokinese hier weiter als bei seiner ersten Aufstellung (1907), wo ich ihn auf den erstgenannten Fall beschränkte. Die neue Fassung scheint mir deshalb geboten, weil es sich nach unseren leutigen Kenntnissen in den beiden Fällen offenbar um ein Geschehen von prinzipiell gleicher Bedeutung handelt.

3) Näheres über diesen Begriff siehe in meiner Publikation von 1907 . 
trationszustand der chromatischen Substanz der Heterochromosomen, welcher stärker oder schwächer ausgesprochen sein kann als bei den gewöhniichen Chromosomen. Besonders charakteristisch ist das namentlich in den Spermiocyten und Spermiden beobachtete Auftreten der Heterochromosomen in Form basophiler Nucleolen, während gleichzeitig die gewöhnlichen Chromosomen langgestreckte Fäden darstellen oder noch stärker aufgelöst sind. Wührend nun das Auffinden als Heteropyknose deutbarer Befunde verbunden mit Heterokinese den Schluss auf Heterochromosomen noch befestigen wird, darf die alleinige Beobachtung basophiler Nucleolen nur mit grosser Forsicht verwertet werden. Es ist nämlich in letzter Zeit mehrfach festgestellt worden, dass in verschiedenen Stadien der Geschlechtszellenbildung basophile Nucleolen vorkommen können, die mit Heterochromosomen nichts zu tun haben (Gutherz, 190 ${ }^{1}$ ) and 1909, Boring, 1907, S. 507). Bei dieser Lage der Dinge werden wir, vor einen basophilen Nucleolus gestellt, nach weiteren in ihm selbst gelegenen Anhaltspunkten suchen, welche die Annabme seiner Chromosomennatur zu stützen vermögen. Solche können sich einmal in gewissen morphologischen Besonderbeiten bieten, die für Chromosomen charakteristisch sind ${ }^{2}$ ) (z. B. Andeutung einer Spirembildung, Ähnlichkeit mit der Gestalt der in Pro- und Metaphase begriffenen gewöhnlichen Chromosomen); sodann in Gestaltveränderungen des Gebildes, die sich als funktionelle deuten lassen, wie solche besonders von $\mathrm{B} \mathrm{uchner}$ (1909, S. $35 \mathrm{lff}$.) bei Orthopteren als für Heterochromosomen charaliteristiscl beschrieben wurden.

\section{Beobachtungen.}

Bei vielen Evertebraten, z. B. den für Heterochromosomenstudien vorzugsweise verwendeten Insekten, sowie bei den niederen

1) $\mathrm{Da}$ von mehreren Seiten meine (1907, S. 509) im Anschluss an $\mathrm{H}$ e nking gegebene Schilderung des basophilen Nucleolus im Oocyten von Pyrrhocoris apterus so aufgefasst wurde, als ob ich denselben mit dem Heterochromosom des Männchens homologisierte, möchte ich betonen, dass es sich nach meiner Überzengung hier um ein basophiles Gebilde handelt, das man nicht als Heterochromosom zu betrachten berechtigt ist.

$\Rightarrow$ Ebensowenig wie die basopbile Färbungsreaktion einen völlig sicheren Schluss auf die chromatische Natur eines Nucleolus zulässt, kann man dieselbe mit Gewissheit aus den morphologischen Eigenschaften eines solchen Gebildes, ohne vollständige Aufklärung seiner Entwicklungsgeschichte, ableiten, da Berghs (1906) an Spirogyra gezeigt hat, dass aus echter Nucleolarsubstanz durchaus chromosomähnliche Gebilde entstehen können. 
Vertebraten (Anamnia) ist die richtige Seriierung der spermiogenetischen Stadien verhälnismïssig leicht zu erreichen, da hier stets zahlreiche Zellen des gleichen oder fast gleichen Stadiums in einer Spermatocyste beisammen liegen und der Inhalt einer Spermatocyste sich in allgemeinen um so weiter entwickelt zeigt, je näher sie dem las deferens gelegen ist. Bei den höheren Vertebraten dagegen, wo der Entwicklungsprozess in Form der sogenannten "Samenbildungswelle" in einer Schraubenlinie längs des Hodenkanalchens abläuft, ist die exakte Seriierung dor Stadien eine sehr schwierige Aufgabe. Dieselbe ist für den Menschen noch nicht gelöst und liegt auch nicht im Plane unserer Untersuchung. Doch darf man sich, wie ich hervorheben möchte, von einer derartigen Behandlung der menschlichen Spermiogenese auci für unsere Spezialfrage mancherlei wertrolle Aufklärung versprechen.

Wir greifen daher die für unser Problem bemerkenswerten Stadien der Spermiogenese ${ }^{1}$ ) zur Betrachtung heraus und beginnen mit der für uns besonders wichtigen Frage nach der Chromosomenzahl. Wie schwierig diese $\mathrm{zu}$ ermitteln ist, geht aus den starken Widersprüchen in der älteren Literatur ${ }^{2}$ ) hervor, besonders eklatant aber aus den im gleichen Jahre gemachten Mitteilungen Guyers und Brancas über die Chromosomenzahl der menschlichen Praespermide. Dieselbe soll nach Branca (1910, S. 8) ungefähr das Doppelte wie im Spermiocyten (also ungefähr 24) betragen. Guyer (1910, S. 227) dagegen findet sie halb so gross wie im Spermiocyten (5 bezw. 7 ), was er auf nochmalige Konjugation der gewöhnlichen Chromosomen zurückführt. Vergleiche ich meine Beobachtungen mit den mir von Insekten her bekannten Verhältnissen, so muss ich konstatieren, dass mir unter zahlreichen Mitosen auch nicht eine Äquatorialplatte zu Gesicht kam, die ich im Falle eines Insektes zur Chromosomenzäblung zugelassen hătte: die Chromosomen liegen meist ausserordentlich dicht oder überdecken einander, da sie niemals streng in einer Ebene liegen; bei sehr dichter Lagerung der Chromosomen ist auch eine ungefähre

1) Die Stadien der Samenbildung werden im folgenden mit Wa ld e yer und v. Leuhossék als Spermiogonien, Spermiocyten (früher Spermatocyten I. Ordnung), Praespermiden (früher Spermatocyten II. Ordnung) und Spermiden bezeichnet.

3) Eine Zusammenstellung derselben findet sich bei Guyer (1910). 
Schätzung ihrer Zahl bäutig ausgeschlossen. ${ }^{1}$ ) Selbst unter den relativ günstigen Bildern, welche die erste Reifungsmitose bietet, fand ich nur zwei Äquatorialplatten, an denen sich eine, jedoch nur annähernde, Zählung vornehmen liess. Die eine derselben ist auf Taf. VI, Fig. 10 abgebildet, Fig. 11 zeigt zwei einzelne Chromosomen der zweiten Platte. In Fig. 10 lassen sich annähernd zwölf Chromosomen zählen; zweifelhaft ist, ob die links oben gelegene Gruppe aus drei oder nur aus zwei Chromosomen besteht. Dasselbe ungefähre Ergebnis hatte die Zählung der Chromosomen der zweiten Äquatorialplatte. Ich kann damit durchaus die Angaben von Duesberg (1906) und Branca (1910) bestätigen. Wenn Guyer mit Bestimmtheit als Chromosomenzahl der ersten Reifungsmitose zwölf angibt, so kann ich ihm hierin nicht folgen. Für die Spermiogonien hebt Guyer die Schwierigkeit der Chromosomenzählung selbst hervor und kann die von ihm mitgeteilte Zahl (22) nur auf wenige Beobachtungen stützen. Über die Chromosomenzahl in den Spermiogonien und Praespermiden bin ich nicht einmal zu einem annähernden Resultat gelangt.

Sind wir somit zulzeit nicht in der Lage, die Chromosomenverhälnisse in der Spermiogenese des Menschen exakt zu verfolgen, so sehen wir uns bei einem Versuch, hier Heterochromosomen nachzuweisen, auf die oben erörterten indirekten Kriterien angewiesen. Während ich in den der Kanälchenwand unmittelbar anliegenden Samenelementen, die grösstenteils wohl als Spermiogonien zu bezeichnen sind, bisher kein auffälliges Gebilde nachweisen kounte, ${ }^{2}$ ) fesselte im Kern des Spermiocyten ein be-

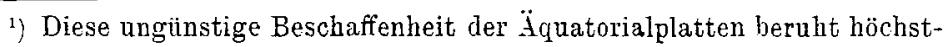
wahrscheinlich auf einer uns im einzelnen unbelannten Wirkung des Fixationsmittels, könnte also vielleicht durch einen Fortschritt der Technik überwunden werden. Auch wäre ein Erfolg durch grosse Austlauer beim Suchen brauchbarer Bilder denkbar, da erfahrungsgemäss sich vereinzelte günstigere Mitosen finden lassen.

3) Zusatz bei der Korrektur: II o n tg o me ry (1911) unterscheidet in einer soeben erschienenen Mitteilung, welche die Entstehung der Sertolischen Zellen des Menschen behandelt, drei Generationen ron Spermiogonien und findet in den Kernen der ersten Generation neben echten Nucleolen basophile Körper, die nach Ansicht des Autors möglicher Weise Heterochromosomen darstellen könnten. Auch ich finde in den Spermiogonienkernen mitunter mehrere basophile Körper. Da aber meist nur echte Nucleolen anzutreffen sind, so handelt es sich in den von mir beobachteten Fällen vielleicht um prophasische Stadien der gewöhnlichen Chromosomen. 
merkenswerter basophiler Nucleolus mein Interesse, ${ }^{1}$ ) der sich bei näherer Untersuchung als konstante Erscheinung erwies. Die Unterscheidung dieses Gebildes ron den gleichzeitig vorhandenen echten, acidophilen Nucleolen (Plasmosomen) gelingt nicht nur durch eine spezifische Färbungsmethode, als welche mir das Biondische Gemisch (nach Fixation mit Zenkerscher Flüssigkeit) diente, ${ }^{2}$ ) sondeln sehr leicht auch bei Anwendung einer indifferenten Färbung, z. B. des Eisenhamatoxylins nach Heidenliain. Die echten Nucleolen im Spermiocyten des Menschen sind numlich fast immer von annähernd genau kugeliger (selten ovaler) Gestalt und dadurch von dem unregelmässiger gestalteten basophilen Nucleolus mühelos zu unterscheiden. Ihre Zahl kann in seltneren Fällen drei betragen; die Zweizahl scheint fast so häufig zu sein, wie das Vorkommen nur eines echten Nucleolus. Sind mehrere derselben vorhanden, so scheint stets nur einer von ihnen die beträchtliche Grösse zu besitzen, die man an dem in der Einzahl vorhandenen echten Kernkörperchen regeimässig konstatiert (Taf. VI, Fig. 1 und 2), die anderen Nucleolen pttegen kleiner zu sein und weisen ebenfalls stets rundliche Form auf. Auch bei Anwendung von Eisenhämatoxylin und weitgehender Differenzierung findet sich fast immer eine färberische Verschiedenheit zwischen echtem und basophilem Nucleolus, die aber der Gesetzmässigkeit entbehrt, indem zwar in der Regel der echte Nucleolus länger die Farbe belält als der basophile, aber auch das umgekebrte Verhalten beobachtet werden kann. Mittels der Fle m ming schen Dreifarbenmethode kann es unter Umständen ebenfalls gelingen. den basophilen von den echten Nucleolen zu differenzieren : er nimmt dann rote Färbung an, während die letzteren blassviolett erscheinen.

Haben wir so bereits färberische und gröbere Gestaltsunterschiede des uns interessierenden Gebildes von den echten Nucleolen kennen gelernt, so gelingt es bei näberer Betrachtung, auch feinere morphologische Besonderheiten an ihm $\mathrm{zu}$ demonstrieren. Hierfür eignet sich vorzüglich die $\mathrm{H}$ eidenhainsche Eisenhämatoxylinfärbung bei möglichst weitgetriebener Differenzierung

1) Von diesem Körper habe ich bereits eine kurze Schilderung in der Sitzung der Gesellschaft naturforschender Freunde zu Berlin vom 16. Mai 1911 gegeben (Sitz.-Ber. 1911, Nr. 5, S. 255).

$\left.{ }^{2}\right)$ Der uns interessierende Körper nimmt hierbei Methylgrün an, daher bezeichnen wir ihn als basophil. Die echten Nucleolen werden durch Säurefuchsin rot gefärbt. 
der Präparate mittels Eisenalauns: auf diese Weise wird der Körper nicht von den Chromatinschleifen verdeckt, was sonst leicht der Fall ist und ein Eindringen in seinen feineren Bau erschwert. Alle Präparate unserer Tafel sind in dieser Weise behandelt; daraus erklärt sich die Blässe der plasmatischen Zellbestandteile und das unscharfe Bild der Chromatinfäden. Der basophile Nucieolus zeigt nunmehr häufig die Struktur eines Doppelstabchens (Fig. 2), mitunter aber, was besonders interessant ist, diejenige einer Vierergruppe (Fig. 3 und 5). Um die auffallende Ähnlichkeit dieser Strukturen mit den sich in der Prophase entwickelnden Chromosomen zu demonstrieren, habe ich letztere Stadien in den Fig. 8 und 9 abgebildet. Die hier gezeigten Vierergruppen, die ihre Vierergruppennatur nicht immer deutlich enthüllen und dann nur Doppelstäbchen bezw. einfache eckige Körperchen darstellen, erinnern ausserordentlich an die verschiedenen Gestalten des basophilen Nucleolus. Leider babe ich diesen aus Mangel an derartigen Stadien noch nicht bis in frühe Prophasen hinein verfolgen können, in denen er sich von den, wie zu erwarten, kurze Doppelfäden repräsentierenden Chromosomen noch klar differenzieren lassen müsste. Ein derartiger Befund würde die Beteiligung des Gebildes an der Mitose sehr wahrscheinlich machen.

Sehr in die Augen springend ist der Umstand, dass der basophile Körper häufig der Kernperipherie genähert erscheint und dann meist mit dem einen Ende seiner langgestreckten Gestalt der Kernmembran aufsitzt, wie dies unsere Fig. 4-7 gut illustrieren. Mit der peripherischen Lagerung ist öfters eine Gestaltveränderung des Körpers verbunden, die häufiger in einer Verjüngung seines aufsitzenden Endes (Fig. 4), mitunter auch in einer Verbreiterung dieser Partie (Fig. 6) besteht. Derartige Bilder, namentlich im Falle der Verjüngung des peripherischen Endes, erinnern stark an die Beschreibungen, welche Buchner (1909) für das Heterochromosom im Spermiocyten mehrerer Ortbopteren gegeben hat und so deutet, dass hier eine Substanzabgabe aus dem Heterochromosom ins Plasma hinein stattfände. Vielleicht sind auch unsere Befunde als funktionelle Veränderungen des basophilen Nucleolus zu deuten.

Über das Verhalten des basophilen Körpers in den verschiedenen Stadien des Spermiocyten kann ich nur wenige Angaben 
machen, da eben eine exakte Seriierung derselben nicht im Plane unserer Untersuchung lag. Im Leptotänstadium lionnte ich den Körper nicht sicher auffinden, allerdings zeigen sich öfters Verdichtungen des Spirems, die aber in der Mehrzahl auftreten und wenig Charakteristisches haben. Ebensowenig konnte ich ihn im synaptischen Kontraktionsstadium demonstrieren, das man zur Unterscheidung von dem ebenfalls zur Synapsis gerechneten Bukettstadium neuerdings als Synizesis bezeichnet, vielleicht wird er aber hier von dem dichten Fadenknäuel verdeckt. Im Bukettstadium kann der Körper dem Pol der Zelle, der durch das Idiozom bezeichnet wird, ganz genühert liegen (Fig. 6), man trifft ihn aber auch entfernt von dieser Stelle an. Hieraus geht bereits hervor, dass er keine konstante Lagebeziebung zum Idiozom (Aunäherung an dasselbe) aufweist, wie Jordan (1911, S. 44) eine solche für den basophilen Nucleolus in sämtlichen Stadien des Spermiocyten von Didelphys virginiana vom Synizesisstadium an beschreibt. Auch in den postsynaptischen Stadien, in welchen man den Körper am leichtesten auffindet, zeigt er keine derartige Lagebeziehung zum Idiozom (vergleiche Fig. 4, wo er fern vom Idiozom liegt). Die vorbin beschriebenen, möglicherweise funktionellen Gestaltsveränderungen des Gebildes finden sich sowohl im Bukettstadium, wie in postsynaptischen Stadien, die Vierergruppenform sah ich bisher nur in postsynaptischen Stadien.

Nachdem ich den basophilen Nucleolus zunächst im Fall A studiert hatte, war es für mich sehr wertvoll, ihn in den Fällen BI-III wiederzufinden und damit sein allgemeines Vorkommen wahrscheinlich zu machen. Er zeigt auch in diesen Fällen die bereits geschilderten Charaktere, wie dies Fig. 5 (Fall BI), Fig. 6 (Fall B II) und Fig. 7 (Fall B III) veranschaulichen. Während Fall BI eine ausgezeichnet lebhafte Spermiogenese aufweist, ist dieselbe in den zur Untersuchung gelangenden Hodenstückchen von Fall B II und B III bedeutend eingeschränkt, wobei sich aber stets zahlireiche Spermiocyten in den verschiedensten Stadien vorfinden. Fall B III weist einige Eigentümlichkeiten auf. Das interstitielle Bindegewebe ist hier sehr stark vermehrt, so dass die Hodenkanälchen wie Inseln erscheinen. Die Spermiocyten (Fig. 7) sind auffallend gross und zeigen gleichfalls sehr grosse echte Nucleolen, die bei der starken Extraktion des Eisenhämatoxylins ganz blass geworden sind. Dadurch tritt 
in diesem Falle der basophile Nucleolus mit besonderer Deutlichkeit hervor. Ob diese Veränderungen als patbologisch zu denten sind, sei dahingestellt.

Mit dem von v. Lenhossék im Spermiocyten der Ratte beschriebenen Intranuclearkörper bat unser basophiler Nucleolus, wie hervorgehoben sei, keinerlei Ähnlichkeit. Dies geht sowohl aus der ursprünglichen Schilderung v. Len hosséks (1898, S. 251 ff.), sowie aus der erneuten Untersuchung des Gebildes durch R e ga ud (1910, S. 325) hervor. Auch mit den von letzterem Autor beim gleichen Objekt beschriebenen safranophilen Körpern (S. 324), die Verdickungen der Chromatinfäden darstellen, bat unser Gebilde nichts zu tun. Ich habe auf diesen Punkt besonders geachtet und kann sicher angeben, dass es sich im Falle des Menschen um einen frei im Kernraume befindlichen Körper handelt.

Wir beschliessen die Darstellung unserer Befunde am Menschen mit der Betrachtung zweier Stadien, die für einen Nachweis von Heterochromosomen von besonderer Bedeutung sind, uns aber in dieser Hinsicht keinen Anbaltspunkt bieten. Guyer hat, ebenso wie andere Autoren (Jordan, Stevens) bei Vertebraten, für den Menschen in der ersten Reifungsmitose Heterokinese (und zwar eines aus zwei Komponenten zusammengesetzten Chromatinkörpers) beschrieben, ${ }^{1}$ ) ferner schildert er einen Dimorphismus der Spermiden als Folgezustand jener Heterokinese (beobachtet an Eisenhämatoxylin-Präparaten). Beide Befunde kann ich nicht bestätigen. Fig. 12-14 zeigen das typische Bild der von der Seite gesehenen Äquatorialplatte der ersten Reifungsmitose: die Chromosomen sind so unregelmässig gelagert (bald liegt eines, bald mehrere dem einen Pol genähert), dass eine Heterokinese, wenn sie tatsächlich vorkäme, sich unserer Feststellung wegen der Unsicherheit der Befunde entziehen müsste. Ich neige aber zu der Auffassung, dass eine Heterokinese überhaupt nicht vorkommt, da sich, wenn auch selten, ziemlich regelmässig ausgebildete Äquatorialplatten finden und diese dann kein einem Pol genähertes Chromosom aufweisen (Fig. 15). Im gleichen Sinne spricht die Betrachtung junger Spermidenstadien (Fig. 16, Schnittdicke $10 \mu$ ). Die meisten der zur Untersuchung kommenden

$\left.{ }^{1}\right)$ Nach Gu ye r liegt hierbei der Heterokinese erfahrende, aus zwei ungleich grossen Chromosomen zusammengesetzte Körper dem einen Spindelpol mehr oder weniger genähert.

Archiv f. mikt. Anat. Bd. 79. Abt. II. 
Kerne zeigen hier grōssere mit Eisenhämatoxylin (bei starker Extraktion) intensiv tingierte Körper. Einen in die Augen fallenden Dimorphismus der Kerne kann ich nicht demonstrieren (die drei Kerne, welche keinen grösseren Körper zeigen, sind nur angescbnitten, enthielten also doch vielleicht einen solchen). ${ }^{1}$ )

\section{Diskussion der Ergebnisse.}

Ein Vergleich unserer Ergebnisse mit denjenigen $G$ uyers zeigt starke Differenzen zwischen beiden. Wenn der amerikanische Autor im Spermiocyten zwei "Chromatinnucleolen", einen grösseren und einen kleineren, (neben gelegentlich vorkommenden anderen kleinen nucleolusartigen Granula) schildert, so spricht alle Wahrscheinlichkeit dafür, dass Guyer, der sich nur der Eisenhämatoxylinmethode bediente. die echten Nucleolen für chromatische angesehen hat, und dass sein grösserer Körper einen echten Nucleolus, der kleinere ebenfalls einen echten Nucleolus oder unseren basophilen Fiörper darstellt. ${ }^{2}$ ) Die Angaben Guyers über Heterokinese und Dimorphismus der Spermidenkerne konnten nicht bestatigt werden.

Anderseits konnten wir ein Gebilde im Spermiocyten demonstrieren, dessen Heterochromosomennatur zweifellos diskutabel erscheint, das aber Guyer offenbar entgangen ist. Für die Deutung dieses Körpers als Heterochromosom sprechen seine basophile Farbungsreaktion seine Ähnlichkeit mit den Chromosomen der Prophase der ersten Reifungsmitose (ror allem die mit unter deut-

1) Herr Dr. H. v. Win i warter (Lüttich), dem ich gelegentlich einer Korrespondenz über meine Befunde beim Menschen berichtete, hatte die Freundlichkeit, mir am 11. Oktober 1911 mitzuteilen, dass er den von uns oben beschriebenen Körper im menschlichen Spermiocyten ebenfalls beobachtet habe. v. Wini warter. der den Körper als Heterochromosom auffasst, lässt ihn Heterokinese eríahren, so dass er schliesslich nur in die Hälfte der Spermien gelangt. Man wird das Erscheinen der Publikation abwarten müssen. ehe man letztere 1 ngabe beurteilen kann.

3) Man könnte an die Möglichkeit denken, diese Differenzen aus einem Rassenunterschied der zur Entersuchung gelangten Individuen zu erklären. da Guyers Material von einem Neger stammte. Ich möchte diese Dentung aber einstweilen für höchst unwahrscheinlich haiten, da Guyer keine spezifische Färbung anwandte und seine Figuren (wenigstens für den Spermiocyten und die erste Reifangsmitose) eine Interpretation im Sinne unserer Befunde durchaus zulassen. 
liche Vierergruppenform), endlich gewisse Gestaltveränderungen des Gebildes, die sich vielleicht als funktionelle Vorgänge auffassen lassen und an Befunde bei sicher erkannten Heterochromosomen anderer Objekte erinnern. Nach all dem Angefübrten darf man meines Erachtens den Körper mit einiger Wahrscheinlichkeit (gewissermaßen heuristisch) in die Kategorie der Heterochromosomen einordnen, ohne sich freilich damit auf sicherem Boden zu bewegen, wie aus unseren methodologischen Vorbemerkungen erhellt.

Gleichwohl sei es gestattet, noch mit einigen Worten darauf einzugehen, welchem Heterochromosomentypus angehörig wir uns den basophilen Körper vorzustellen bätten. Da eine Heterokinese nicht nachzuweisen ist, so werden wir zu der Annahme geführt, dass es sich um ein Chromosomenpaar handele, dessen Fomponenten gleich gross sind und im Laufe der Reifungsteilungen gleichmässig auf die Spermiden verteilt werden. Damit stimmt sein mitunter beobachtetes Auftreten in Form der Vierergruppe, die ja von jeher als Prototyp eines bivalenten Chromosoms gilt.

Unsere Annahme findet eine Stütze an Beobachtungen, die an einem anderen, ebenfalls den Vertebraten angehörigen Untersuchungsobjekt, der Hauskatze, vorgenommen wurden. Von Winiwarter und Saintm on t (1909, S. 234 ff.) beschreiben hier im Oocytenkern einen Körper, der durch seine in gewissen Stadien auftretende Gestalt (Doppelstäbchen) an unseren basophilen Nucleolus erinnert und den sie als Heterochromosom auffassen. Er zeigt zwar nicht die Lageeigentümlichkeiten und Gestaltverănderungen des beim Menschen beobachteten Gebildes, dürfte aber etwas Verwandtes darstellen. Ich habe nun an Sammlungspräparaten des Berliner anatomisch-biologischen Instituts, die mir freundlichst zur Verfügung gestellt wurden, im Spermiocyten des Katers einen ganz ähnlichen Körper finden können (Textfig. A und B), über dessen etwaige spezifische Färbungsreaktion ich allerdings noch nichts aussagen kann, da die Präparate mit Eisenbämatoxylin gefürbt waren. Übereinstimmend ist auch der Umstand, dass, wie bei der Katze, im Synapsisstadium die Doppelstäbchennatur noch nicht hervortritt (Fig. A). Dieser Befund, der allerdings noch der Bestätigung mittels einer spezifschen Färbungsmethode und der Ergänzung durch Verfolgung des 
Körpers in der Spermiogenese bedarf, spricht dafür, dass es sich hier, wenn überhaupt um Chromosomen. um ein Chromosomenpaar handelt. das in beiden Geschlechtern Heteropyknose erfährt.

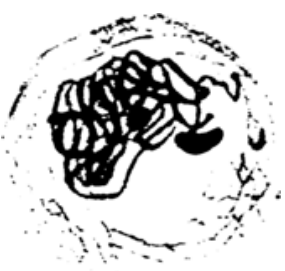

Fig. 1 .

Spermiocyt des Katers im Synapsisstadium.

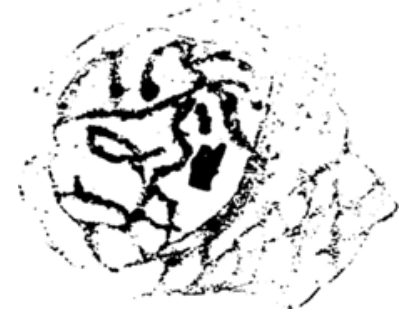

Fig B.

Spermiocyt des Katers in postsynaptischem Stadium.

Zusatz bei der Korrektur: Eine inzwischen Forgenommene Untersuchnog des in Carnoyscher Flüssigkeit (abs. Alkohol 6, Chloroform 3. Eisessig 1) tixierten Katerhodens mittels des B i o n $d$ i schen Farbengemisches änderte meine Luffussung des in den Fig. A und B abgebildeten Körpers wesentlich. Er erweist sich nämlich hierbei in beiden Stadien als ausgesprochen acidophil, indem er sich mit Säurefuchsin leuchtend rot färbt, während das Chromatin Jethylgrün annimmt: es handelt sich also um einen echten Nucleolus, der durch seine Gestalt ein Heterochromosom in Heteropyknose vortänscht. Der ron $v$. Winiwarter und Sainmont im Oocyten der Katze beobachtete und als Heterochromosom aufgefasste Körper wird hierdurch in seiner Deutang äusserst zweifelhaft, da die Autoren keine spezifische Färbung vornahmen and mit der Fle m ming schen Dreifarbenmethode Blanfärbung des Gebildes erzielten; während nach meinen Erfahrungen nucleolenartige chromatische Körper bei dieser Methode stets rot tingiert werden. Der Fall der Katze muss daber aus jeder Diskussion über Heterochromnsomen ausscheiden, solange er nicht mittels einer einwandfreien spezifischen Lethode untersucht ist. Unsere Befunde im Spermiocyten des Katers, die einen echten Vacleolus in durchaus chromosomähnlicher Gestalt demonstrieren, mahnen auch za besonderer Vorsicht bei der Deutung der am Spermiocsten des Yenschen gemachten Beobachtungen.

Es sei noch bemerkt, dass die Annahme eines Heterochromosomenpaares mit gleicher Grösse der Komponenten, wie wir es uns beim Menschen vorzustellen bätten, nicht die Möglichkeit einer Beziehung der betreffenden Chromosomen zur Geschlechtsdifferenzierung ausschliesst. E. B. Wilson hatte angegeben, dass derartige Fälle sich durch Übergänge verbunden eng an solche an=chliessen können, die im mănnlichen Geschlecht ein Heterochromosomenpaar mit ungleicher Komponentengrösse 
(verbunden mit Dimorphismus der Spermiden) aufweisen. Hat Wils on (1911 a) auch neuerdings gezeigt, dass der von ihm zuerst hierfür in Anspruch genommene Fall der Hemipterenart Nezara hilaris doch eine ganz geringe Grössendifferenz der Komponenten des Chromsomenpaares besitzt, so hält er gleichwohl das Vorkommen derartiger Fâlle für möglich und nimmt hierbei eine physiologische Differenz zwischen den beiden gleich grossen Komponenten an.

\section{Zusammenfassung.}

1. Im Spermiocyten des Menschen findet sich neben einem bis drei echten Nucleolen ein basophiler Nucleolus, der auf Grund seiner feineren Struktur (Doppelstabchenbezw. Vierergruppenform) und gewisser als funktionell zu deutender Gestaltverănderungen mit einiger Wabrscheinlichkeit den Heterochromosomen einzuordnen ist.

2. Da weder Heterokinese noch ein Dimorphismus der Spermidenkerne nachzuweisen sind, so ist das hypothetische Heterochromosom als Chromosomenpaar mit gleich grossen Komponenten zu betrachten, wofür auch seine mitunter zu beobachtende Vierergruppenform spricht.

3. Die Angaben Guyers, der für den Menschen typische Geschlechtschromosomen beschreibt, konnten nicht bestätigt werden.

Berlin, 9. Dezember 1911.

\section{Literaturverzeichnis}

B a l t zer, F.: Die Chromosomen von Strongylocentrotus lividus und Echinus microtuberculatus. Arch. f. Zellforsch., Bd. 2, 1909.

B erg h s, J.: Le noyau et la cinèse chez le Spirogyra. La Cellule, T. 23, 1906.

Boring, A. M.: A study of the spermatogenesis of 22 speeies of the Membracidae etc., with especial reference to the behavior of the odd chromosome. Journ. of exp. Zool., Vol. IV, 1907.

B ranca, A.: Caractères des deux mitoses de maturation chez l'homme. Cpts. rds. Assoc. des Anatom., 12. Réun., Bruxelles 1910.

$\mathrm{Buchner}$, P.: Das accessorische Chromosom in Spermatogenese und Ovogenese der Orthopteren etc. Arch. f. Zellforsch., Bd. 3, 1909.

D uesberg, J.: Sur le nombre des chromosomes chez l'homme. Anat. Anz., Bd. 28, 1906. 
Gnlick, A.: Über die Geschlechtschromosomen bei einigen Nematoden etc. Arch. f. Zellforsch., Bd. 6, 1911.

G u ther z, S.: Zur Kenntnis der Heterochromosomen. Arch. f. mikr. Anat., Bd. 69,1907 .

Derselbe: Wird die Annahme einer Beziehung zwischen Heterochromosomen und Geschlechtsbestimmung durch das Studium der Gryllus-Oogenese widerlegt? Sitz. - Ber. Ges. naturforsch. Freunde, Berlin 1909, Nr. 9.

Derselbe: Über den gegenwärtigen Stand der Heterochromosomen-Forschung, nebst Bemerkungen zum Problem der Geschlechtsdifferenzierung. Ibid. 1911, Nr. 5 .

G u yer, M. F.: The spermatogenesis of the domestic Guinea. Anat. Anz. Bत. $3 \pm 1909$.

Derselbe: The spermatogenesis of the domestic chicken. Ibid. 1909.

Derselbe: Accessory chromosomes in man. Biol. Bull., Vol. 19, 1910.

Jord a n, H. E.: The spermatogenesis of the Opossum (Didelphys virginiana) with special reference to the accessory chromosome and the chondriosomes. Arch. f. Zellforsch., Bd. 7, 1911.

Lenhossek, M. v.: Untersuchungen über Spermatogenese. Arch. f. mikr. Anat., Bd. is, 1898.

Hontgomery, Th. H.: Differentiation of the human cells of Sertoli, Biol. Bull.. Vol. 21, 1911.

Morrill, Ch. V.: The chromosomes in the oogenesis, fertilization and cleavage of Coreid Hemiptera. Biol. Bull., Vol. 19, 1910.

Rega ud, Cl.: Etudes sur la structure des tubes séminifères et sur la spermatogenèse chez les mammifères. Arch. d'Anat. microsc., T. 11, 1910.

Stevens, N. M.: Preliminary note on heterochromosomes in the Guineapig. Biol. Bull., Vol. 20, 1911.

Wilson, E. B.: Studies on cluromosomes. VII. A review of the chromosomes of Nezara; with some more general considerations. Journ. of Morphol., Vol. 22, 1911 a.

Derselbe: The sex chromosomes. Arch. f. mikr. Anat, Abt. II, Bd. 77, 1911 b.

Winiwarter: H. v. et $\mathrm{Sa}$ inmont, G.: Nouvelles recherches sur l'ovogénèse et l'organogénèse de l'ovaire des mammifères (chat), chap. IV. Arch. d. Biol., T. 24, 1909. 


\section{Erklärung der Abbildungen auf Tafel VI.}

Sämtliche Figuren sind bei $\mathrm{Z}$ e is s' Apochromat $2 \mathrm{~mm}$, Kompens. Okular 12 in der Höhe des Objekttisches gezeichnet (Vergr. 1500) und stellen Stadien der menschlichen Spermiogenese dar. Wo nichts anderes bemerkt ist, stammen die Präparate von Fall A. Fixation: Flem ming s starkes Gemisch. Färbung: Eisenhämatoxylinmethode nach $\mathrm{H}$ e i $\mathbf{d}$ e $\mathbf{n} \mathbf{h}$ a in (weitgehende Differenzierung der Präparate).

Fig. 1. Spermiocyt in postsynaptischem Stadium. Echter Nucleolus.

Fig. 2. Dasselbe. Links oben basophiler Nucleolus (Doppelstäbchen), rechts unten echter Nucleolus.

Fig. 3. Dasselbe. Basophiler Nucleolus in Vierergruppenform.

Fig. 4. Dasselbe. Basophiler Nucleolus mit dem verjüngten Ende der Kernmembran aufsitzend. Idiozom entfernt vom basophilen Nucleolus gelegen.

Fig. כ. Dasselbe. Fall B I. Basophiler Nucleolus in Vierergruppenform.

Fig. 6. Spermiocyt im Bukettstadium. Fall B II. Basophiler Nucleolus mit dem verbreiterten Ende der Kernmembran aufsitzend.

Fig. 7 Spermiocyt in postsynaptischem Stadium. Fall B III. Basophiler Nacleolus.

Fig. $\&$ und 9. Prophase der ersten Reifungsmitose. Chromosomen zum Teil in deutlicher Vierergruppenform.

Fig. 10. Metaphase der ersten Reifungsmitose, Äquatorialplatte vom Spindelpole aus gesehen. Chromosomenzahl annähernd 12.

Fig. 11. Zwei einzelne Chromosomen einer ähnlichen ت̈quatorialplatte.

Fig. 12-15. Metaphase der ersten Reifungsmitose, in seitlicher Ansicht.

Fig. 16. Gruppe junger Spermiden. 


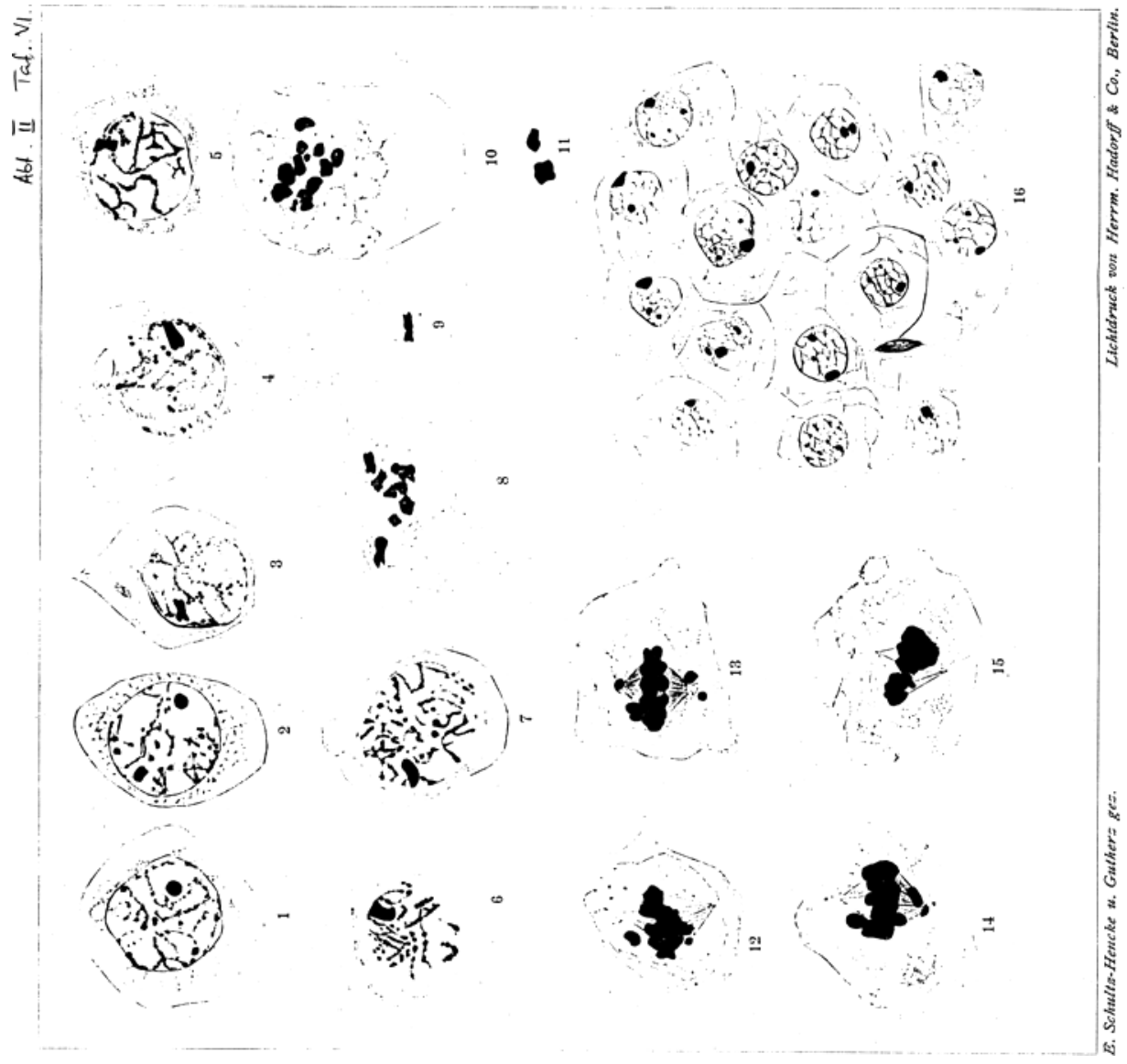

